\title{
On the Galois and flat cohomology of unipotent algebraic groups over non-perfect fields
}

\author{
By Nguyêñ Quôć ThăŃG*) and Nguyêñ Duy TÂN \\ Institute of Mathematics, 18 - Hoang Quoc Viet \\ 10307 - Hanoi, Vietnam \\ (Communicated by Heisuke HironakA, M. J. A., June 14, 2005)
}

\begin{abstract}
We prove some new results on the triviality and the finiteness of the Galois and flat cohomology of unipotent algebraic group schemes over non-perfect (and in particular, also local and global function) fields. Some applications are also given to certain local - global principles.
\end{abstract}

Key words: Galois cohomology; flat cohomology; unipotent groups.

1. Introduction. It is well-known by a result of Rosenlicht [Ro1, Ro2] that if $G$ is a smooth connected unipotent algebraic group defined over a perfect field $k$ then the group structure of $G$ is very simple: there is a normal series of $k$-subgroups of $G$, with each factor isomorphic over $k$ to the additive group $\mathbf{G}_{a}$ (cf. also [Bo]). In particular, its first Galois cohomology, which will be denoted by $\mathrm{H}^{1}(k, G):=\mathrm{H}^{1}\left(\operatorname{Gal}\left(k_{s} / k\right), G\left(k_{s}\right)\right)$, where $\operatorname{Gal}\left(k_{s} / k\right)$ denotes the absolute Galois group of $k$, is trivial (see, e.g. [Se]). However this is no longer true if $k$ is non-perfect. First Rosenlicht [Ro1, Ro2], then Tits [Ti], Raynaud [Ra], Demazure and Gabriel [DG] and Kambayashi, Miyanishi and Takeuchi ([KMT]) were able to show certain unusual properties of unipotent groups over non-perfect fields. They also established basic properties of unipotent algebraic groups over non-perfect fields. In [Se], Chap. III, Serre constructed certain function field $k$ and unipotent $k$-group $G$, such that the first Galois cohomology $\mathrm{H}^{1}(k, G)$ of $G$ is non-trivial (cf. also Raynaud [Ra]). Furthermore, Oesterlé developed in [Oe] a comprehensive arithmetic theory of unipotent groups over global fields and, in particular, showed that the nature of unipotent groups over non-perfect fields is quite non-trivial. One example given there showed that this first Galois cohomology group may be even infinite for some locally compact field of characteristic $p>0$ (a completion of a global function field). In general, there are many open interesting (and difficult) questions regarding the arithmetic and geome-

2000 Mathematics Subject Classification. Primary 11E72; Secondary 18G50, 20G10.

*) Regular Associate of the Abdus Salam I. C. T. P. try of unipotent algebraic groups over non-perfect fields. For example, there is a close connection with the Néron models theory of unipotent algebraic groups ([Oe], [BLR]). In particular it is natural and interesting to inquire about the Galois cohomology of unipotent groups in this case. Here we are interested in the finiteness and certain local - global principles especially in the case where $k$ is a local or global function field of characteristic $p>0$. We assume that, unless otherwise stated, all algebraic groups considered here are linear algebraic groups in the classical sense, i.e., absolutely reduced (or smooth) as in [Bo], [Oe].

2. Main results. We proceed in this paper, by basing on some main results from the theory of unipotent groups over non-perfect fields of [DG], [KMT], [Oe], [Ro1, Ro2], [Ra], [Ti] to investigate certain finiteness properties of Galois (and also flat) cohomology of unipotent groups over non-perfect fields, generalizing the results mentioned above by Serre, Raynaud and Oesterlé, and also investigate some related local - global principles over global function fields.

Let $k$ be a global field, $S$ a finite non-empty set of non-equivalent valuations of $k$. We denote by $k_{v}$ the completion of $k$ at a valuation $v$, and by $A(S)$ the ring of (truncated) $S$-adèles of $k$. If $P:=$ $P\left(T_{1}, \ldots, T_{n}\right) \in K\left[T_{1}, \ldots, T_{n}\right]$ is a polynomial with coefficients in a field $K$ and in $n$ variables $T_{1}, \ldots, T_{n}$, one says that $P$ is universal over $K$, if $P\left(K^{n}\right)=K$. We say that $P$ is additive, if for any two elements $x \in K^{n}, y \in K^{n}$ we have $P(x+y)=P(x)+P(y)$. If this is the case, $P$ is the so-called a $p$-polynomial, where $p=$ char. $K$. Denote by $p^{m_{i}}$ the highest de- 
gree of $T_{i}$ appearing in $P$ with the coefficient $c_{i} \in$ $K^{*}$. Then the sum $\Sigma_{1 \leq i \leq r} c_{i} T_{i}^{p^{m_{i}}}$ is called the principal part of a $p$-polynomial $P$. For $p>0$, we denote by $X_{t}$ the closed $k$-subgroup of $\mathbf{G}_{a}^{2}$ defined by the equation $y^{p}=x+t x^{p}$. By $\mathrm{H}^{*}$ we denote the Galois cohomology and by $\mathrm{H}_{f p p f}^{*}$ we denote the flat cohomology, which is isomorphic to the Galois cohomology in the case of smooth group schemes.

The main results of this note are the following announcements, the detailed proof of which will be published elsewhere.

Theorem 1. Let $k$ be a non-perfect field of characteristic $p$ with a non-trivial discrete valuation. Let $G$ be a non-trivial (not necessarily smooth) commutative unipotent $k$-group scheme of dimension $\leq$ 1 , the connected component $G^{\circ}$ of which is not $k$ isomorphic to an extension of $\mathbf{G}_{a}$ by an infinitesimal $k$-group scheme. Assume in addition that if $p=$ 2 then $G^{\circ}$ is neither $k$-isomorphic to an extension of $\mathbf{G}_{a}$, nor of the subgroup $X_{t}$ defined above, by an infinitesimal group scheme. Then the flat cohomology $\mathrm{H}_{\text {fppf }}^{1}(k, G)$ is infinite. In particular, for any global field $k$ of positive characteristic and for any non-trivial commutative $k$-unipotent group scheme $G$ of dimension $\leq 1$ with $G^{\circ}$ not $k$-isomorphic to an extension of $\mathbf{G}_{a}$, by an infinitesimal k-group scheme, the cohomology group $\mathrm{H}_{f p p f}^{1}(k, G)$ is infinite.

This theorem generalizes some known results (cf. Serre [Se], Ch. III, Raynaud [Ra], Exp. XVII, Sec. 5.9, Oesterlé [Oe], Ch. IV, Sec. 2.2). It is interesting to note that the situation is quite different over local function fields as the following examples show.

Exemples. 1) Let $k$ be a global function field of characteristic $p=2, t \in k \backslash k^{2}$, and let $v$ be a nontrivial discrete valuation of $k$. Then for $G=X_{t}$, we have $\left|\mathrm{H}^{1}\left(k_{v}, G\right)\right|=2$, and $\mathrm{H}^{1}(k, G)$ is infinite.

2) Let $k=\mathbf{F}_{q}(t), k_{v}=\mathbf{F}_{q}((t)), q=p^{n}, p>2$, and let $G$ be the cloased subgroup of $\mathbf{G}_{a}^{2}$ d'efined by the equation $x_{0}^{p}+t x_{1}^{p}+\cdots+t^{p-1} x_{p-1}^{p-1}=x_{p-1}$. Then $\left|\mathrm{H}^{1}\left(k_{v}, G\right)\right|=p$, and $\mathrm{H}^{1}(k, G)$ is infinite.

3) Let $k=\mathbf{F}_{q}(t), k_{v}=\mathbf{F}_{q}((t)), q=p^{n}, p>$ 2 , and let $G$ be the closed subgroup of $\mathbf{G}_{a}^{2}$ defined by the equation $x_{0}^{p}+t x_{1}^{p}+\cdots+t^{p-1} x_{p-1}^{p-1}+x_{p-1}=$ 0 . Then $\left|\mathrm{H}^{1}\left(k_{v}, G\right)\right|=1$ (resp. $p$ ) if $n$ is odd (resp. even), $\mathrm{H}^{1}(k, G)$ is infinite.

Concerning the finiteness and the triviality of the Galois and flat cohomologies of the groups $G$ above, we have the following principle of local - global type, which relates the finiteness (or the triviality) of the cohomology with the property of being closed for orbits in the topology induced from that of $A(S)$.

Theorem 2. Let $k$ be a global function field of characteristic $p>0$, and let $G$ be a connected smooth unipotent group scheme defined over $k$.

a) The first Galois cohomology set $\mathrm{H}^{1}(k, G)$ is trivial if and only if for all valuation $v$ of $k, \mathrm{H}^{1}\left(k_{v}, G\right)$ $i s$ trivial and for some (hence any) $k$-embedding of $G$ into a semisimple (resp. unipotent) smooth simply connected $k$-group $H$, then with natural action of $H$ on $H / G$, the orbits of $H(k)$ are closed in $(H / G)(k)$ in the $A(S)$-topology of $(H / G)(k)$ for any finite set $S$ of valuations of $k$.

b) The first Galois cohomology set $\mathrm{H}^{1}(k, G)$ is trivial if it is finite and for some (hence any) $k$ embedding of $G$ into a semisimple (resp. unipotent) smooth simply connected $k$-group $H$, then with natural action of $H$ on $H / G$, the orbits of $H(k)$ are closed in $(H / G)(k)$ in the $A(S)$-topology of $(H / G)(k)$ for any finite set $S$ of valuations of $k$.

In particular, in the case the group $G$ is defined by an additive polynomial, we have the following local - global principle.

Theorem 3. Let $P$ be a separable additive (i.e. $p$-)polynomial in $n$ variables with coefficients in a global function field $k$ of char. $p>0$.

a) $P$ is universal over $k$ if and only if $P$ is universal over $k_{v}$ for all valuations $v$ of $k$, and $P\left(k^{n}\right)$ is $A(S)$-closed in $k$ for all finite set $S$ of valuations of $k$.

b) Assume that $n=2$. Then $P$ is universal over $K$ if and only if it is so over all $k_{v}$.

In order to establish these results, besides the known results by [DG], [Oe], [Ra], [Ro1, Ro2], [Ti], and various preliminary ones, we need the following key results.

Theorem 4 ([TT]). For any field $k$ with any finite non-empty set $S$ of (non-equivalent) valuations of $k$ and any smooth unipotent algebraic group $G$ defined over $k$, the localisation map of Galois cohomology

$$
\phi_{S}: \mathrm{H}^{1}(k, G) \rightarrow \prod_{v \in S} \mathrm{H}^{1}\left(k_{v}, G\right)
$$

is surjective.

Theorem 5. Let $k$ be a field of characteristic $p>0, v$ a discrete non-trivial valuation of $k$, and let $G$ be a smooth commutative unipotent group defined by a separable p-polynomial $P$ in $r$ variables with the 
principal part

$$
P_{0}=\sum_{1 \leq i \leq r} c_{i} T_{i}^{p^{m_{i}}}, c_{i} \in k^{*} .
$$

We set $m=\min \left\{m_{1}, \ldots, m_{r}\right\}$ and suppose that $r<p^{m}$ and that $v\left(c_{1}\right), \ldots, v\left(c_{r}\right)$ are pairwise distinct modulo $p^{m}$. Then the cohomology set $\mathrm{H}^{1}(k, G)$ is infinite.

(In fact, the condition of the discreteness of the valuation stated above is not essential.) As an immediate consequence of the results obtained, we have the following partial answer to a question mentioned by Russell in [Ru].

Corollary. Let $k$ be a non-perfect field of characteristic $p>0$ with a non-trivial discrete valuation. Then for any natural number $n, k$ has cyclic extensions of degree $p^{n}$.

Acknowledgements. We would like to thank T. Kambayashi and J. Oesterlé for some useful correspondences and T. Ekehdal for interesting conversations related to the paper. The first author greatly acknowledge the supports given by Fund. Res. Prog. Vietnam, The Abdus Salam ICTP (Italy) and Institut des Hautes Études Scientifiques (France) while working on the paper.

\section{References}

[Bo] A. Borel, Linear algebraic groups, 2nd ed. Springer, New York, 1991.
[BLR] S. Bosch, W. Lütkebohmert and M. Raynaud, Néron models, Springer, Berlin, 1990.

[DG] M. Demazure and P. Gabriel, Groupes algébriques, tome I, Masson \& Cie, Éditeur, Paris, 1970.

[KMT] T. Kambayashi, M. Miyanishi and M. Takeuchi, Unipotent algebraic groups, Lecture Notes in Math., 414, Springer, Berlin, 1974.

[Oe] J. Oesterlé, Nombres de Tamagawa et groupes unipotents en caractéristique $p$, Invent. Math. $\mathbf{7 8}$ (1984), no. 1, 13-88.

[Ra] M. Raynaud, Groupes algébriques unipotents, Extensions entre groupes unipotents et groupes de type multiplicatif, Exp. XVII, in Schémas en groupes, SGA 3 (2), Lecture Notes in Math., vol. 152, Springer, New York, 1970, pp. 532-631.

[Ro1] M. Rosenlicht, Some rationality questions on algebraic groups, Ann. Mat. Pura Appl. (4) 43 (1957), 25-50.

[Ro2] M. Rosenlicht, Questions of rationality for solvable algebraic groups over nonperfect fields, Ann. Mat. Pura Appl. (4) 61 (1963), 97-120.

[Ru] P. Russell, Forms of the affine line and its additive group, Pacific J. Math. 32 (1970), 527-539.

[Se] J.-P. Serre, Cohomologie galoisienne, 5th ed., Springer, Berlin, 1994.

[TT] N. Q. Thăńg and N. D. Tan, On the surjectivity of localization maps for Galois cohomology of unipotent algebraic groups over fields, Comm. Algebra 32 (2004), no. 8, 3169-3177.

[ Ti ] J. Tits, Lectures on algebraic groups, Yale Univ., New Haven, 1967. 